\title{
CLUSTER BASED ROUTING PROTOCOLS IN WSN SENSOR
}

\author{
Kanak Prabha Lila Ramani ${ }^{1}$, Dr. Abhishek Badholia ${ }^{2}$ \\ ${ }^{1,2}$ Computer Science and Engineering, MATS University, Raipur,India \\ ${ }^{1}$ kanakp31@gmail.com
}

\begin{abstract}
Over the past few years we can observe the WSNs or Wireless Sensor Network applications in various fields increasing immensely. The energy efficiency, network lifetime and clustering process prime goal is the working network's optimization is the focus of many of the routing algorithm. Keeping in mind the network homogeneity, for network performance reinforcement we suggest instead of single path to use multiple paths. For WSNs, Reinforcement Intelligence Routing Protocol (RIRP)[1]. In the multihop wireless sensor networks an efficient and effective method for security improvements local monitoring has worked well [2]. But taking in consideration the power consumption in the current practice of local monitoring is costly. For ensuring longlived operations in the sensor network reinforcement intelligent routing protocol is critical [3]. For ensuring both the aspects improvement in security and long-lived operations, the development of mechanism that is effective and incorporated with the Reinforcement protocol is an open problem. With the help of local monitoring to solve this issue, section of the traffic going in and out of its neighbors is supervised by each node to keep a check on any suspicious behavior like unlikely long delays in packet forwarding [4]. To integrate the existing reinforcement protocol of the network and without any niggardly in the consumption of energy in the local monitoring with the help of a protocol [5]. In comparison to other protocols in this protocol the region of instability starts later. At a constant rate the nodes of the RIRP or Reinforcement Intelligent Routing Protocol dies. Few problems such as cluster head selection process, network lifetime and network stability are evaluated and worked in the technique proposed here [6]. To reduce the overload consumption as much as possible the nodes switches in between the active and sleep mode.
\end{abstract}

Keyword: new dataset; artificial intelligence; sensor; natural language processing; fake detector; machine learning.

\section{Introduction}

In present generation just like all technologies and sciences, WSNs or Wireless Sensor Network is also encountering possibilities in the betterment of the already existing solutions, aiming at the approaches that are cost efficient and time saving, leading to the development of new concepts and approaches in the particular field. In the intended domain comprehensive and quick access to these methods are given by Survey studies[7]. Moving forward with this motive in mind and considering WSNs energy consumption management and controlling by the impact of cluster processing, hence in terms of methodology to give an expensive assessment by centralizing the focus to the cluster-based multi-hop routing protocol and clustering. To evaluate the characteristics of various methodologies some parameters are presented in this survey. Then in 4 categories the perspective of the methodology is classified from the studied methods as[9]:

i. Fuzzy-based approaches

ii. Classical approaches

iii. Hybrid metaheuristic and fuzzy-based approaches and iv. Metaheuristic - based approaches.

To estimate the method in every categories of classification according to the type of methodology parameters and criteria are presented; therefore, on the basis of the methodologybased parameters and cluster-based parameters evaluation of each method of the class is done and discussed eventually [11]. In the process to motivate audiences and give an useful and accurate information, this development, despite of giving an useful assignment, for the method of examination intend to present a new approach by the consideration of methodology based characteristics like of each method examined input and output, constraints and capabilities, the reason of using the algorithm, in the method the type of algorithms used, etc[12]. The given survey can be proved helpful for researchers as the basis of the fast understanding of deficiencies and shortcomings in this field for any future coming further investigations [13].

In a form of a clusters or certain groups division of sensor is done, in the WSNs clustering, every clusters and group has cluster head. After an event or periodically to the $\mathrm{CHs}$ the levant information is transferred by the sensor in each cluster [14]. Straightto the multi-hop or BS way these information is transmitted by the CHs. There are many benefits of clustering few of them are like clustering can prevent redundancy of exchange messages or communication bandwidth [15]. 
(a)Stabilization of the network topology by Clustering at the sensor level and because of the only $\mathrm{CHs}$ node interaction reduces community overhead.

(b)In the network implementation of optimized management technology can be done by clustering.

Additionally, with the mechanism of clustering, in the reduction in consumption of energy and that in turn leads to the network lifetime increase by routing for sending their data is a very important characteristic. Hence the in WSNs, the design for routing protocol is challenging as it includes the limitations in the network energy efficiency[16]. In designing of routing protocol few of the effective characteristics are provided here:

Constraints in the energy consumption: in WSNs each sensor node are provided with a battery that has limited energy that makes the work like sending, receiving and computing into a very challenging task[17] and a heavy dependency on the lifetime of the nodes on the sensor.

Nodes deploy: in the area of network the sensor node distribution is dependent on program and no monitoring of humans, hence routing protocol performance is affected [18].

Capabilities of nodes: various functions and roles can be carried out by sensor nodes like aggregation, sensing, relaying and mixing the data in accordance to the type of strategy; energy consumption is done by all these roles and in the designing of these protocols is considered as challenge [19].

Aggregation of data: duplicate events may be sensed by the adjacent sensor node of the network. To stop any kind of redundancy of data from getting sent to BS, proper aggregation methods can be considered [20].

Tolerance of Faults: because of the wireless connections there are uncertainties in these networks hence studying various dynamic topologies and mobility patterns should be considered [21].

In WSNs for designing effective energy protocol many parameters should be taken in account, in consideration to the problems mentioned above. Whereas the sensor network research fields are very expensive and because their field of applications are getting expended, they are becoming more widespread [22]. Additionally, mechanism of routing and clustering, in the other areas of the sensor network are also being used like trajectory designing, mobile sink trajectory and on the even fields so then the sensor network[23]. To have a clustering design and effective routing the performance is affected by different factors, to recognize the shortcoming and deficiency, the designer needs to target the perfect and accurate information of these methods[24]. At this extensive field with the goal of receivingan improved accurate examination with a battery that is limited as well as not nonrechargeable in the clustering-based routing protocol is the main focus of this survey[25]. To make a proper usage of
WSNs several protocols, algorithms and methods have been introduced having different purposes[26]. There is a need of a method that on the basis of algorithms used, methods used, methods characteristics of every part, etc. From the methodology perspective examination survey, given in this field sand protocols methods exists in large numbers; the survey should be such that despite of the giving the complex judgments[27], gives the designers shorter path for the research and the methods details of this fields is provided in a general prospective. Considering the methodology prospective design of the survey is such that it[28]at the cluster-based routing protocol field as the starting point. With the aim of identification of the shortages and deficiencies to be facilitated in the WSNs, cluster-based routing protocol field giving outlines of the details of goals, methods, algorithms, parameters, etc. Is the main focus of the survey[29].

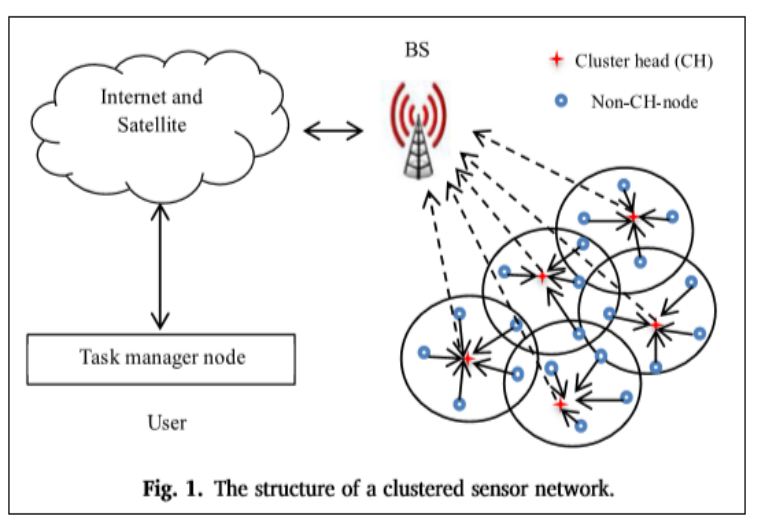

Figure 1 The clustered sensor network structure.

The clustered WSNs structure is shown in the figure 1 . In the record of the abbreviation of the words used in this paper and the summarized definitions in brief is provided [30]. The cluster based multi hop Routing and clustering surveys that are done before hand are reviewed in this survey. In the survey along with the descriptions, classification factor of the presented parameters is provided [31].Having the aim of focusing on the objectives with it's equivalent presentation by the function of the cluster based multi hop routing protocols and Clustering, along with the comparing with the classification protocol examination is done in the form of table and is discussed simultaneously. The conclusions and directions for future study related to this topic are provided [32].

\section{Contributions of this survey}

From the methodology perspective in WSNs, cluster-based routing protocol is the main focus of this survey. Taking into consideration the examination protocols methodology details along with the clustering methods-based properties [33]. Few parameters are provided in these two basic classifications to compare the cluster-based routing protocol [34]. Two 
classification of clustering is done into: micro and macro, this classification is done for better evaluation of methods and also to give a partial and general view of the clustering protocol process [35]. Here the categories methodology is divided as Hybrid metaheuristic and Metaheuristic-based approaches , Fuzzy-based approaches and Classical approaches.

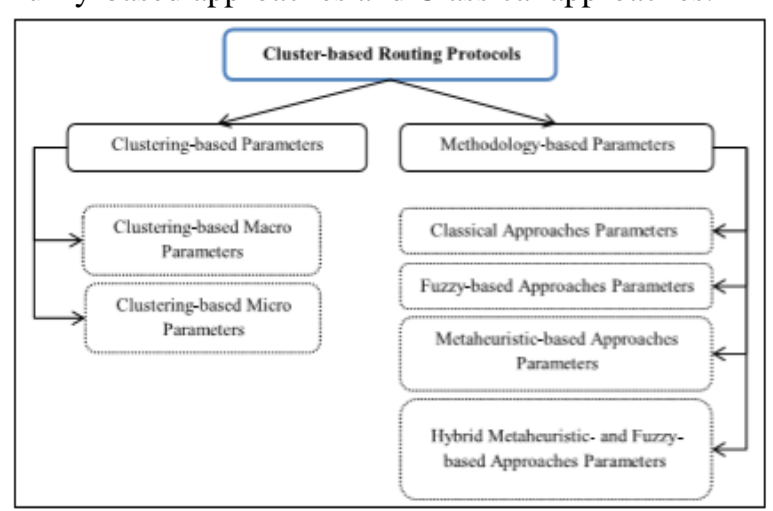

Figure 2 Cluster based routing protocol

classifications based approaches

In figure 2 brief of the structure of the complete classification explained in the survey is represented. Summary of the survey contribution is shown here:

1. Especially for the field of cluster-based routing protocol evaluation, a new approach and perspective was presented, for reviewing the existing algorithm and protocol presented [37].

2. In accordance to the point of view that is methodologybased a new classification was presented.

3. For every classification category new methodology-based parameters and approaches are presented [38].

4. Based on the clustering micro parameter and macro parameters that are also clustering based provisions in clusterbased routing protocol for giving a partial and general view on the clustering process is considered [39].

5. For serving cluster-based routing protocol as a starting point, in WSNs for cluster-based routing protocol, a extensive and comprehensive evaluation is provided so as to accelerate and simplify the methodology, protocols apprehension and the prospective of the methodology defect identification[40].

In optimization algorithm and technique of network lifetime maximization in network aggregation technique, QoS assurance congestion control multi-object optimization privacy and on similar subject there are many surveys in WSNs [41]. Having said that, in accordance to the prime aim of the understanding in the following sequence of the various survey are discussed that centralize routing protocol, clustering protocols and cluster based multi hop routing protocols. In wireless sensor network routing protocol survey is presented; three general section hierarchical, data centric and based on the local section, categorization is donein different methods and each category method is discussed. In this survey consideration of the classification of methods into three general sections, as well as the quality of services modeling and network flow method is done [41].Clustering algorithm for WSNs evaluation paper was presented by Younis and Abbasi in the year 2007, they presented one of the first evolution paper and survey on this topic. For the Clustering protocol,clustering attributes and convergence time are the two basic classifications presented in their survey [42]. In the clustering attributes terms division of methods are dome in sections of three that includes the properties of clustering, properties of cluster and process of clustering. Whereas,into two variables as well as two fixed parts the methods are divided in the terms of convergence time [43]. In accordance to the classification being proposed, their main aims and the methods are summarized, with the parameters like overlapping of cluster, cluster stability, convergent cerate, etc. In clustering algorithm to evaluate the section mechanisms of $\mathrm{CHs}$, are the evaluation methods that are presented in a survey. Into four categories classification of all methods is done in this survey as follows adaptive, deterministic, hybrid clustering and combined metric[44]. Two subsectionsare present in the adaptive category diving it to theadaptive probabilistic and parameter probabilistic that are fixed. On the basis of classification and sub classification final evaluation of the clustering algorithm is done.

\section{Criteria and classification factors}

Characterization of the classification parameter is done in this section. At first, division of clustering-based parameter is done into two clustering-based micro parameters and clusteringbased macro parameters. Clustering methods microcharacteristics and macro-characteristics are included in these two categories [45]. In accordance to the classification presented introductions of the methodology-based parameters are done in algorithms and methods on the bases on the methodology used [46].

i. Clustering-based parameters: here the clustering evolution parameters are presented by using two methods for the clustering model macro-characteristics and microcharacteristics analysis called as clustering based macro parameters and clustering based micro parameters.

ii. Clustering-based macro parameters: in this section discussion on Clustering based macro parameters is done. On evolution methods general information is provided by these parameters like method general objective, method of $\mathrm{CH}$ selection and hierarchical structure[15].

iii. Hierarchical: hierarchical network structure can be used to minimize the long-distance data as the network management and control is facilitated by it. Congestion is prevented by this Provisionals. In the method of hierarchical clustering both the normal nodes and $\mathrm{CH}$ nodes are putted in different levels here direct connection to BS usually by the $\mathrm{CH}$ nodes[31]. Selection of the two type of $\mathrm{CHs}$ are done using few methods. To transmit the information to from level to another level of the CHs is done by the first level which in turn is responsible 
to transmit information to the BS. By the hierarchy three levels characterization of three methods is done. In many ways to the BS transmission of the information is done in a multi-level way or multi hop. Amongst the CHs of network sometimes such structure is used and some other time among the cluster nodes. In the reviewed ways to find the number of levels created is the parameter aim. Hence the numbers of levels are parameter specific criteria are two, three and multi-level hierarchical parameters, respectively. In the network structure methods that consist of more than three levels employment to Multilevel hierarchy structure is used. Selection method of $\mathrm{CH}$ : the general method of selecting $\mathrm{CHs}$ is described in brief here, using various methods in a way that also serves for the audience benefit. In the reviewed method $\mathrm{CHs}$ selection significant approach[46].

IV. Objective: there are various objectives of routing and clustering methods and algorithms like reducing the number of control messages, maintaining coverage network, removing hot spots problem, utilizing sleeping schemes, avoiding collision, decreasing delay, increasing connectivity, reducing energy consumption, maximizing network lifetime, stability of network topology, load balancing, data aggregation/fusion, fault-tolerance and scalability. Some of these objectives are the main objectives like increasing the lifetime of the network, network topology stability, load balancing, data aggregation/fusion, fault tolerance and scalability [47]. out of the mare consideration the left-out objectives are the secondary objectives that are used to achieve the primary goal. In different methods of clustering the primary objectives are of more importance than the secondary objectives. In the clustering based micro parameters more in depth details of the objective can be found. Clustering based micro parameters is described on this section and method of clustering characteristics that are discussed below. Overview of this parameter is shown in the figure 5 .

Application: the method and algorithm for information collection by $\mathrm{CHs}$ from the normal nodes and BS transmission is categorized into time driven, event driven and query driven. In the query driven method. In query-driven method, query to nodes is sent by BS, simultaneously the information is transmitted back to BS by node, to the query response. In event-driven method, the collected to the BS the transmission of the data is done by sensor, in accordance to a particular threshold or at an occurrence of an event. In time-driven method, remission of data is done to periodically to the gateway or BS by the sensor nodes[48].

Cluster size the size of the cluster is controlled or uncontrolled. To analyze algorithm or method the issue of criterion is done to make sure if the designer has paid attention in sin a cluster numbers of members or size controlling of the cluster. Determination of the size of the cluster A is possible by keeping in mind the distance by a B sort for solving the hot stop issue. In such condition, closer cluster heads are smaller and farther cluster heads are larger cluster. For cluster rang determination, size of the cluster may also be based on the cluster algorithm density.

Intra-cluster communicationin few methods there is direct communication in between a relevant cluster head and a sensor . It's advantageous to use intra-cluster communication, in the method in which small number of $\mathrm{CHs}$ are present and where from the cluster heads member nodes are far away. Therefore, in this criterion the consideration of the parameters is done as one hop or multi hop of evaluation-

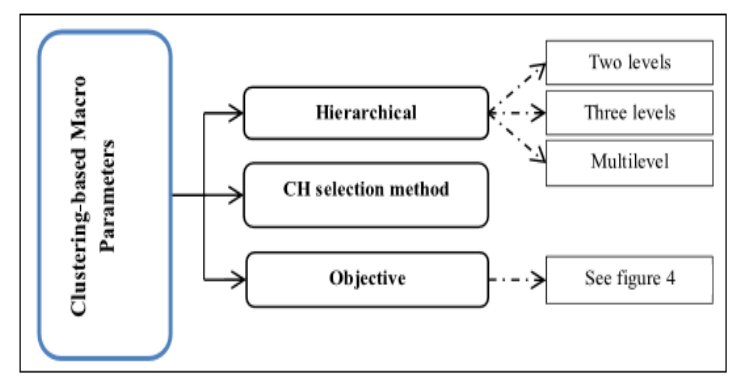

Figure 3 In Wireless Sensor Network the clustering based macro parameters.

V. Inter-cluster communication: in between $\mathrm{CHs}$ and a BS there can be a direct connection or one hop connection. The $\mathrm{CHs}$ of the middle modes in a multi hop way can also be connected. For WSNs can be a good option for a multi hop mechanism can be good option, if in the area sensor nodes in large numbers are present or if with a limited range receivers or transmitters is equipped on the sensor nodes. Method: either centralized or distributed the method of cluster algorithm can be. In such method the task performing process can be centralized mechanisms and hybrid distributed. There is a possibility with the direct BS or with the help BS, the routing phase is centralized whereas the method is self organization or distributed in the phase of clustering and it is distributed by nodes. In the algorithm entire process is adopted by the parameters that is used for mechanism analysis. - Mobility of nodes: either be mobile (moving) or motionless (nonmoving) are Normal nodes and CHs. They can move in limited range if they are considered as mobile. - Node types: algorithms can either use heterogeneous nodes (which are different in equipment and source) if algorithm apply such node from heterogeneous sensor nodes $\mathrm{CHs}$ are usually selected or homogeneous node or normal (in respect to Determination of criterion whether a process of interest use same chainsmtore place the cluster head role playing nodes. In certain methods periodical replacement of the $\mathrm{CHs}$ is done. Once the $\mathrm{CHs}$ energy level reaches threshold that is predetermined is reaches or after predetermined duration of time, they are replacing in other methods. Using the energy threshold method, the energy consumption of the network is tried to be unified in this method[49]. 


\section{In accordance to the methodology cluster based routing protocol classification}

To meet the needs like energy reduction, on which high dependency of these network is by clustering WSNs are benefitted. In different classifications various algorithms and methods for this purpose have been presented according to section 3 . To category present method, methodology view new classification as: Fuzzy-based approaches, Classical approaches, Hybrid metaheuristic and fuzzy-based approaches and Metaheuristic-based approaches . Because of the no present usage of the neutral network method they removed in this area, an overview of algorithm and methodology is analysis is presented in every section. They are talked in brief over here.

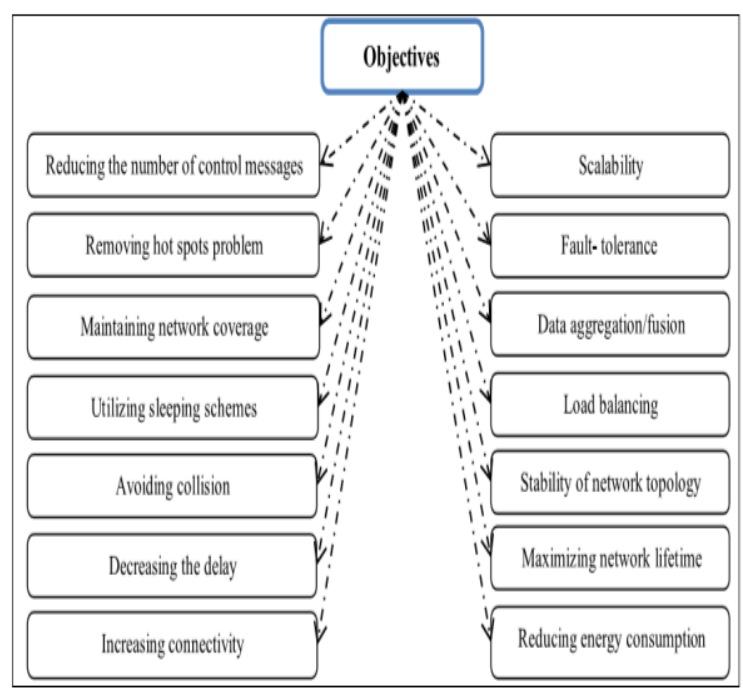

Figure 4 Objectives of Clustering.

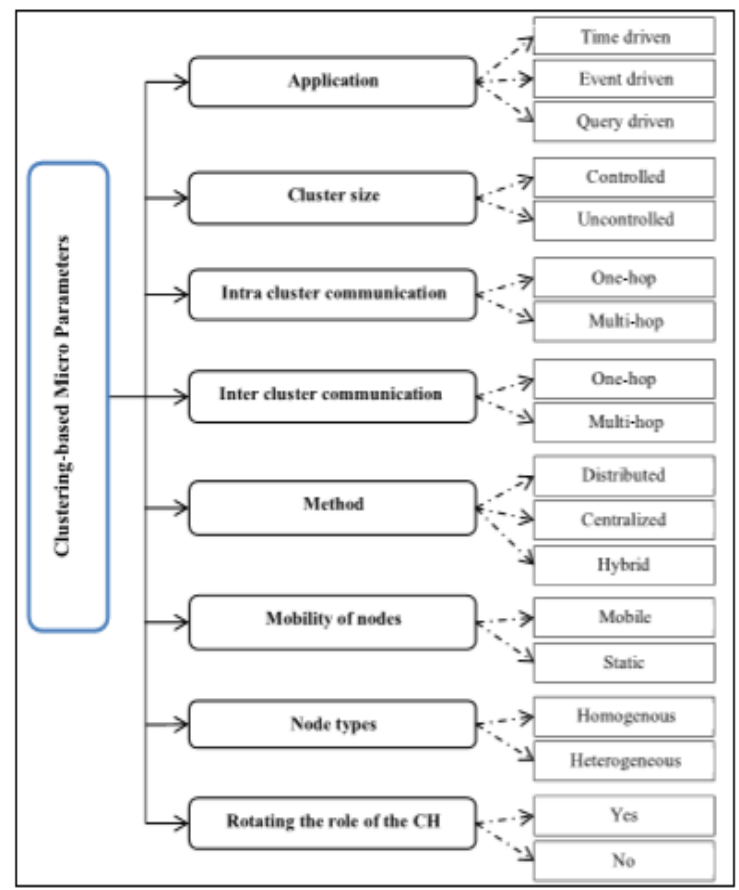

Figure 5 In Wireless Sensor Network Clustering based micro parameters.

\section{PERFORMANCE EVALUATION}

We assess the already existing protocol in WSN called as LEACH and our proposed protocol performance and compare them.

\section{A. Simulation Setting}

We simulated our protocol with MATLAB, to apprise our protocols performance. Here consideration of a field that is $100 \mathrm{~m} \times 100 \mathrm{~m}$ with the wireless sense of 100 nodes is distributed randomly. Deploying a gateway node in the center of the sensing field is done. At the very far distance of the sensing field BS is located. Once the deployment is done, both the BS and gateway node is stationary. 4000 bits packet size is considered here. With the LEACH protocol our protocol is compared. For the comparison of our protocol with LEACH, wireless channel interference and the effect caused by signal collision are over looked. Radio parameters are present in the table 1 .

\section{B. Performance Parameters}

The performance metric is presented in this subsection. Here we evaluate three parameters given below:

1. Lifetime of network: The time of the network operation starts till the last node dies.

2. Throughput: for performance throughput evaluation number of the packets node send in every round is compared with the $\mathrm{BS}$ receiving the number of the packets.

3. Residual energy: analyzing energy consumption of nodes in every round, consideration of the networks residual battery energy is done. Network life graceful degradation is ensured by residual energy.

\section{Simulation result and analysis}

We show simulation result in this subsection. We compare with LEACH by running extensive simulation. Details of each metric is given in the subsection.

1) Lifetime of network: results of network lifetime is shown in figure 3. After consuming 0.5 joule energy nodes are considered dead. Longest network lifetime is obtained in $\mathrm{M}$ GEAR protocol as amongst the nodes energy is well distributed. Dividing network into logical region is further subdivided in cluster the two of them. Among the sensor nodes energy consumption is balanced by M-GEAR topology. As in LEACH, the networks stability duration ends quickly the nodes dies. It is not very clear in LEACH that the perdestined have uniform distribution throughout the field of network. Hence it has the possibility of the chosen Cluster Heads are concentrated at the networks particular places. Therefore few of the nodes does not have environs. Network lifetime Interval plot with $99 \%$ confidence interval is shown in the figure 3. The M-GARE protocol results are different statically and performs good. 


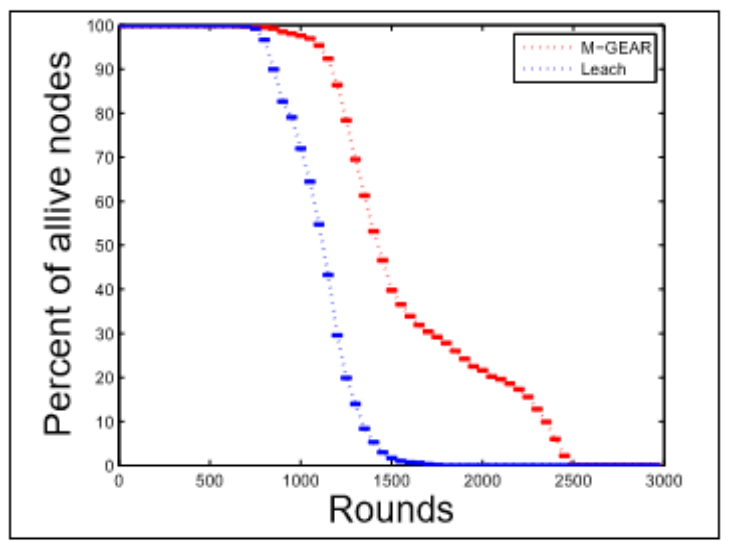

Figure 6 Network lifetime Interval plot - Analysis.

2. Throughput: with extensive simulation assess of average packets sent to $\mathrm{BS}$ is done. Increased throughput by the simulation result of the M-GEAR protocol illustration. In figure 4 interval plot of LEACH and M-GEAR is clearly shows the effectiveness of both the protocols. For the calculation of the throughput, free communication of the Cluster Heads is assumed . 5 times more than LEACH the simulation result is shown here. With the sensor nodes the data is sent directly to the gateway. Similarly transmission of data to the BS is done by bodes near. Less transmission energy is consumed in both region in sensor node. Hence for longer period nodes stay alive, this contributes to more packet transmission to BS.

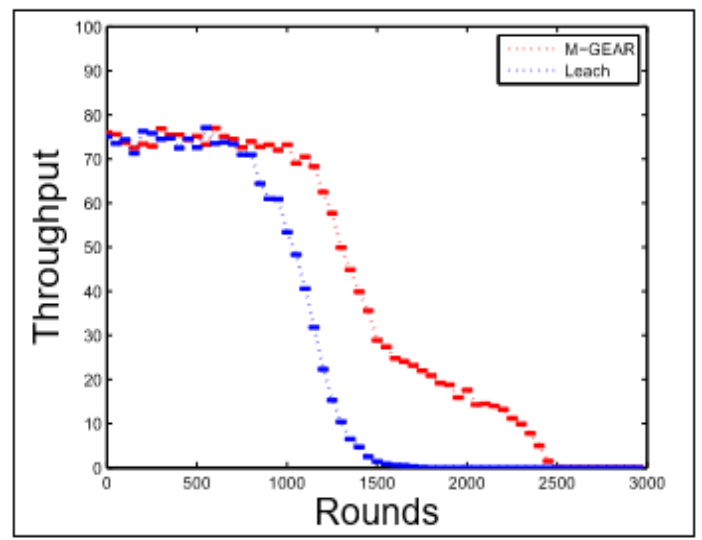

Figure 7 Throughput of the Interval plot- Analysis.

3) Residual energy- per round network's average residual energy is shown in figure 5. Assuming that the energy of 0.5 joule is present in the node. 50 joules is the overall or say the total energy that is present in the 100 nodes. Minimum energy consumption is shown by M-GEAR protocol in comparison of the LEACH. In the energy consumption terms per round our protocol clearly outperforms the LEACH can be seen in the figure 5. Minimum energy consumption is assured by high probability of $\mathrm{CHs}$ in every region and at node deployment of gateway.

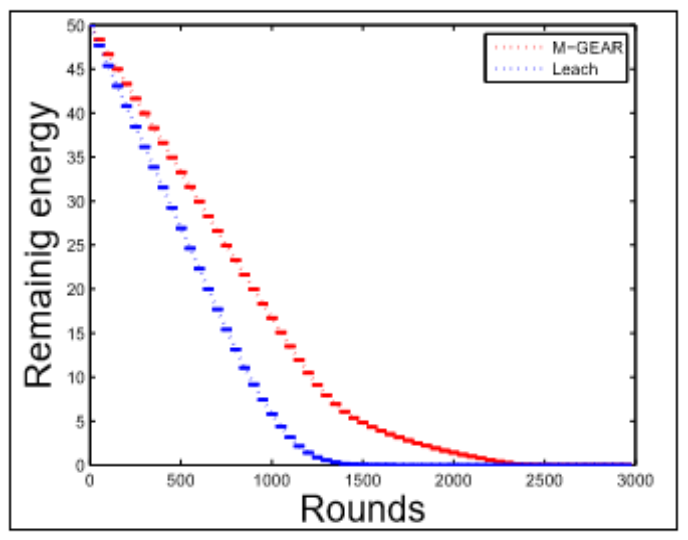

Figure 8 Remaining energy Interval plot analysis

\section{CONCLUSION AND FUTURE WORK}

We have mentioned here an efficient energy multi hop routing protocol by using gateway for the sensor network's energy consumption to be minimized. Into local region the network is divided here. Different communication hierarchy of each region. Using direct communication topology in two regions and in other two more sub divisions to the clusters and uses multi hop hierarchy. Independent of another region each node selects themselves the cluster head of its area. In the network better distribution of $\mathrm{CHs}$ is encouraged in this technique. Depicts that the protocol proposed by us shows better performance than $\mathrm{LEACH}$. We three performance metric in this work: throughout, residual energy and network lifetime. Study of ETX link metric will be done in future and implementation of this metrics will be done in this scheme as demonstrated and implemented.

\section{References}

[1]. Tarun Dhar Diwan, Dr.H.S.Hota, Dr. Siddhartha Choubey "Multifactor Authentication Methods: A Framework for Their Selection and Comparison" accepted for publication in International Journal of Future Generation Communication and Networking Vol. 13, No. 3, (2020), pp. 2522-2538, ISSN: 22337857 (Web of Science).

[2]. Tarun Dhar Diwan, Dr.H.S.Hota, Dr. Siddhartha Choubey "A Novel Hybrid Approach for Cyber Security in IoT network Using Deep Learning Techniques" accepted for publication in International Journal of Advanced Science and Technology ISSN:2394-5125, ISSN: 2005-4238 (Scopus indexed Journal).

[3]. Tarun Dhar Diwan, Dr.H.S.Hota, Dr. Siddhartha Choubey entitled "Development of Real Time Automated Security System for Internet of Things (IoT)" accepted for publication in International Journal of Advanced Science and Technology Vol. 
29, No. 6s, (2020), pp. 4180 - 4195, ISSN: $2005-$ 4238 (Scopus indexed Journal).

[4]. Tarun Dhar Diwan, Dr.H.S.Hota, Dr. Siddhartha Choubey "A Proposed Security Framework for Internet of Things: An Overview" presented in international Conference held on 20-22 December,2019, MTMI, Inc. USA in Collaboration with at amity Institute of Higher Education, Mauritius.

[5]. Tarun Dhar Diwan, Dr.H.S.Hota, Dr. Siddhartha Choubey "Control of Public Services for Public Safety through Cloud Computing Environment" presented in international Conference held on 04-05 January,2020, Organized by Atal Bihari Vajpayee University, Bilaspur in association with MTMI, USA and sponsored by CGCOST, Raipur (C.G), India.

[6]. Tarun Dhar Diwan, Dr.H.S.Hota, Dr. Siddhartha Choubey "A Study on Security and Data Privacy issues of IoT based Application in Modern Society" presented in international Conference held on 04-05 January,2020, Organized by Atal Bihari Vajpayee University, Bilaspur in association with MTMI, USA and sponsored by CGCOST, Raipur (C.G), India.

[7]. Fakhrosadat Fanian, MarjanKuchaki Rafsanjani Cluster-based routing protocols in wireless sensor networks: A survey based on methodology, ournal of Network and Computer Applications 142 · May 2019 DOI: 10.1016/j.jnca.2019.04.021.

[8]. Q. Nadeem; M.B. Rasheed; N. Javaid; Z.A. Khan ; Y. Maqsood ; A. Din, M-GEAR: GatewayBased Energy-Aware Multi-hop Routing Protocol for

WSNs,23December,2013,DOI: 10.1109/BWCCA.

\subsection{5}

[9]. Khanna, A., Gupta, D., Bhattacharyya, S., Snasel, V., Platos, J., \& Hassanien, A. E. (Eds.). (2020). International Conference on Innovative Computing and Communications. Advances in Intelligent Systems and Computing. doi:10.1007/978-981-15-1286-5.

[10]. Afsar, M.M., Tayarani-Najaran, M.H., 2014. Clustering in sensor networks: a literature survey. J. Netw. Comput. Appl. 46, 198-226.

[11]. Agrawal, T., Kushwah, R.S., 2015. Layered clustering routing protocol with overlapping cluster heads in WSN. In: Proceedings of theCommunication Systems and Network
Technologies (CSNT), Gwalior, India, pp. 244-248. April. 4-6.

[12]. Akkaya, K., Younis, M., 2005. A survey on routing protocols for wireless sensor networks. Ad Hoc Netw. 3 (3), 325-349.

[13]. Akyildiz, I.F., Su, W., Sankarasubramaniam, Y., Cayirci, E., 2002. Wireless sensor networks: a survey. Comput. Network. 38 (4), 393-422.

[14]. Alaybeyoglu, A., 2015. A distributed fuzzy logicbased root selection algorithm for wireless sensor networks. Comput. Electr. Eng. 41, 216-225.

[15]. Alsaafin, A., Khedr, A.M., Al Aghbari, Z., 2018. Distributed trajectory design for data gathering using mobile sink in wireless sensor networks. AEU-International Journal of Electronics and Communications 96, 1-12.

[16]. Amgoth, T., Jana, P.K., 2015. Energy-aware routing algorithm for wireless sensor networks. Comput. Electr. Eng. 41, 357-367.

[17]. Amin, R., Islam, S.H., Biswas, G., Khan, M.K., Kumar, N., 2018. A robust and anonymous patient monitoring system using wireless medical sensor networks. Future Gener. Comput. Syst. 80, 483 495.

[18]. [18] Amis, A.D., Prakash, R., Vuong, T.H., Huynh, D.T., 2000. Max-min D-cluster formation in wireless ad hoc networks. In: Proceedings of the Annual Joint Conference of the IEEE Computer and Communications Societies (INFOCOM), Tel Aviv, Israel, pp. 32-41. Mar. 26-30.

[19]. [19] Arjunan, S., Pothula, S., 2017. A survey on unequal clustering protocols in Wireless Sensor Networks. Journal of King Saud UniversityComputer and Information Sciences 29 (4), 428448.

[20]. Arora, V.K., Sharma, V., Sachdeva, M., 2016. A survey on LEACH and other's routing protocols in wireless sensor network. Optik-International Journal for Light and Electron Optics 127 (16), 6590-6600.

[21]. Avci, B., Trajcevski, G., Tamassia, R., Scheuermann, P., Zhou, F., 2017. Efficient detection of motion-trend predicates in wireless sensor networks. Comput. Commun. 101, 26-43.

[22]. Bagci, H., Yazici, A., 2013. An energy aware fuzzy approach to unequal clustering in wireless sensor networks. Appl. Soft Comput. 13 (4), 1741-1749.

[23]. Bagherzadeh, J., Samadzamini, M., 2009. A clustering algorithm for wireless sensor networks based on density of sensors. In: Proceedings of the International Conference on Advances in Mobile Computing and Multimedia (MoMM), Kuala Lumpur, Malaysia, pp. 594-598. Dec. 14 - 16. 
[24]. Baranidharan, B., Santhi, B., 2016. Ducf: distributed load balancing unequal clustering in wireless sensor networks using fuzzy approach. Appl. Soft Comput. 40, 495-506.

[25]. Biswas, S., Das, R., Chatterjee, P., 2018. Energyefficient connected target coverage in multi-hop wireless sensor networks. Ind. Inter. Innov. Sci. Eng. Technol. 11, 411-421.

[26]. Borges, L.M., Velez, F.J., Lebres, A.S., 2014. Survey on the characterization and classification of wireless sensor network applications. IEEE Commun. Surveys Tutorials 16 (4), 1860-1890.

[27]. 21.Butun, I., Morgera, S.D., Sankar, R., 2014. A survey of intrusion detection systems in wireless sensor networks. IEEE Commun. Surveys Tutorials 16 (1), 266-282.

[28]. Cengiz, K., Dag, T., 2015. Low energy fixed clustering algorithm (LEFCA) for wireless sensor networks. In: Proceedings of the International Conference on Computing and Network Communications (CoCoNet), Trivandrum, India, pp. 79-84. Dec.16-19.

[29]. Cengiz, K., Dag, T., 2016. Improving energyefficiency of WSNs through LEFCA. Int. J. Distributed Sens. Netw. 12 (8), 8139401.

[30]. Cengiz, K., Dag, T., 2016. Multi-hop low energy fixed clustering algorithm (M-LEFCA) for WSNs. In: International Symposium on Telecommunication Technologies (ISTT), Kuala Lumpur, Malaysia, pp. 31-34. Nov. 28-30.

[31]. Cengiz, K., Dag, T., 2018. Energy aware multi-hop routing protocol for WSNs. IEEE Access 6, 26222633. Chang, R.S., Kuo, C.J., 2006. An energy efficient routing mechanism for wireless sensor networks. In: Proceedings of the International Conference on Advanced Information Networking and Applications (AINA), Vienna, Austria, vol. 2, pp. 308-312. April 18- 20.

[32]. Chen, D.R., 2015. A link-and hop-constrained clustering for multi-hop wireless sensor networks. Comput. Commun. 72, 78-92. Curry, R.M., Smith, J.C., 2016. A survey of optimization algorithms for wireless sensor network lifetime maximization. Comput. Ind. Eng. 101, 145-166.

[33]. Dargie, W., Poellabauer, C., 2010. Fundamentals of Wireless Sensor Networks: Theory and Practice. John Wiley \& Sons. Dehghani, S., Pourzaferani, M., Barekatain, B., 2015. Comparison on energyefficient cluster based routing algorithms in wireless sensor network. Pro. Com. Sci 72, 535542.

[34]. FCE Deosarkar, B.P., Yadav, N.S., Yadav, R., 2008. Cluster head selection in clustering algorithms for wireless sensor networks: a survey. In: Proceedings of the International Conference on Computing, Communication and Networking, (ICCCN), VI, USA, pp. 1-8. Dec. 18-20.

[35]. Dhurgadevi, M., Devi, P.M., 2018. An analysis of energy efficiency improvement through wireless energy transfer in wireless sensor network. Wireless Pers. Commun. 98 (4), 3377-3391.

[36]. Ehsan, S., Hamdaoui, B., 2012. A survey on energy-efficient routing techniques with QoS assurances for wireless multimedia sensor networks. IEEE Communications Surveys \& Tutorials 14 (2), 265-278.

[37]. Eldhose, E.K., Jisha, G., 2016. Active cluster node aggregation scheme in wireless sensor network using neural network. Pro. Techno. 24, 1603-1608.

[38]. Enami, N., Moghadam, R.A., Ahmadi, K.D., 2010. A new neural network-based energy efficient clustering protocol for wireless sensor networks. In: Proceedings of the International Conference on Computer Sciences and Convergence Information Technology (ICCIT), Seoul, Korea (South), pp. 4045. Nov. 30-2 Dec.

[39]. Faheem, M., Gungor, V.C., 2018. Energy efficient and QoS-aware routing protocol for wireless sensor network-based smart grid applications in the context of industry 4.0. Appl. Soft Comput. 68, 910-922.

[40]. Fan, X., Du, F., 2015. Shuffled frog leaping algorithm based unequal clustering strategy for wireless sensor networks. Appl. Math. 9 (3), 1415 1426.

[41]. Fanian, F., Kuchaki Rafsanjani, M., 2018. Memetic fuzzy clustering protocol for wireless sensor networks: Shuffled frog leaping algorithm. Appl. Soft Comput. 71, 568-590.

[42]. Fanian, F., Kuchaki Rafsanjani, M., KhatibiBardsiri, V., 2016. A survey of advanced LEACH-based protocols. Inter. J. Eng. Infor. Comm. 7 (1), 1-16.

[43]. Fasolo, E., Rossi, M., Widmer, J., Zorzi, M., 2007. In-network aggregation techniques for wireless sensor networks: a survey. IEEE Wireless Comm. 14 (2).

[44]. Fathany, M.Y., Adiono, T., 2015. Wireless protocol design for smart home on mesh wireless sensor network. In: Proceedings of the International Symposium on Intelligent Signal Processing and Communication Systems (ISPACS), Nusa Dua, Indonesia, pp. 462-467. Nov. 9-12.

[45]. Fei, Z., Li, B., Yang, S., Xing, C., Chen, H., Hanzo, L., 2017. A survey of multi-objective optimization in wireless sensor networks: metrics, algorithms, 
and open problems. IEEE Communications Surveys \& Tutorials 19 (1), 550-586.

[46]. Ferdoush, S., Li, X., 2014. Wireless sensor network system design using Raspberry Pi and Arduino for environmental monitoring applications. Pro. Com. Sci 34, 103-110.

[47]. Frye, L., Cheng, L., 2009. Topology management for wireless sensor networks. In: Misra, S., Woungang, I., Misra, S.C. (Eds.), Guide to Wireless Sensor Networks. Springer, London.

[48]. Al-Karaki, J.N., Kamal, A.E., 2004. Routing techniques in wireless sensor networks: a survey. IEEE wireless communications 11 (6), 6-28.

[49]. Abbasi, A.A., Younis, M., 2007. A survey on clustering algorithms for wireless sensor networks. Comput. Commun. 30 (14), 2826-2841. 